\title{
Textured Rulers and Arcs
}

\author{
Chris Shaw, \\ Department of Computer Science, \\ University of Regina, 3737 Wascana Parkway, \\ Regina, Saskatchewan, S4S 0A2, CANADA \\ Phone (306) 585-4071 Fax (306) 585-4745 \\ cdshaw@acm.org \\ May 11, 1997
}

\begin{abstract}
Texture maps representing linear and arc measurement scales provide a visually accessible set of measurements without adding scene clutter or auxiliary measurement widgets.
\end{abstract}

KEYWORDS: Interactive Techniques, Rendering Algorithms, Texture Mapping, Virtual/Interactive Environments. 


\section{Textured Rulers and Arcs}

In designing or examining free-form surfaces or polygonal objects, there is often a need to display linear dimensions of the object, especially when the object must fit with other objects, as in the case of mechanical or industrial design. The standard technique of underlaying axisaligned grids for measurement is convenient only in an orthographic view. In a perspective scene, a shadow of the object can be orthographically projected onto surrounding walls that contain a grid [1]. One measurement at a time can also be provided by an inquiry function, where the user temporarily attaches a measurement widget to the desired objects, but the widget will obscure the objects. For general angle measurement, almost the only choice is a 3 -point measurement widget that will obscure part of the scene while reading out the angle.

The approach suggested in this sketch is simply to mark the surface directly with texturemapped measurement tools. In a similar technique, SGI has a demo that maps a regular grid texture onto a bicubic patch to show its $(u, v)$ parameter space. The main advantage with this approach is that input clutter is eliminated, because there are no widgets to avoid clicking. Instead, the surface is painted with measurements which can be turned on or off as a rendering attribute. Output clutter is also reduced because the measurements occupy the identical space as the object being measured, meaning that the measurements cannot occlude other objects in the scene. Because images are textured onto the surface, potentially hundreds or thousands of measurements are available simultaneously to the user - the user simply has to look at the points of interest to learn the dimension of interest.

To measure the edge lengths of polygon $P$, a bilevel ruler texture is applied to each edge. An inner inscribed polygon is generated in the plane of $P$, and for each edge a 4-sided ruler quad is generated to hold the texture. For example, a triangle would be divided into an inscribed triangle and 3 ruler quads.

For angle measurement, the bilevel arc texture map is used, which is essentially a protractor with heavy tickmarks at 10 degree intervals (Figure 1). For each angle of the polygon to be measured, the axis of this protractor image (at the center of the bottom edge) is placed at the vertex, with the line marked $\mathbf{0}$ located along the edge lying counter-clockwise from the vertex. To apply the arc at each vertex, the polygon must be cut into subpolygons with the arc texture applied to each, as shown schematically in figure 1. For a triangle, each subpolygon is a quadrilateral that is made up of the main triangle's vertex, the midpoint of each incident edge and the centroid of the main triangle. Both arcs and rulers can be 
displayed at the same time, in which case the texturing occurs using the inscribed vertex values for the edge and centroid calculations.
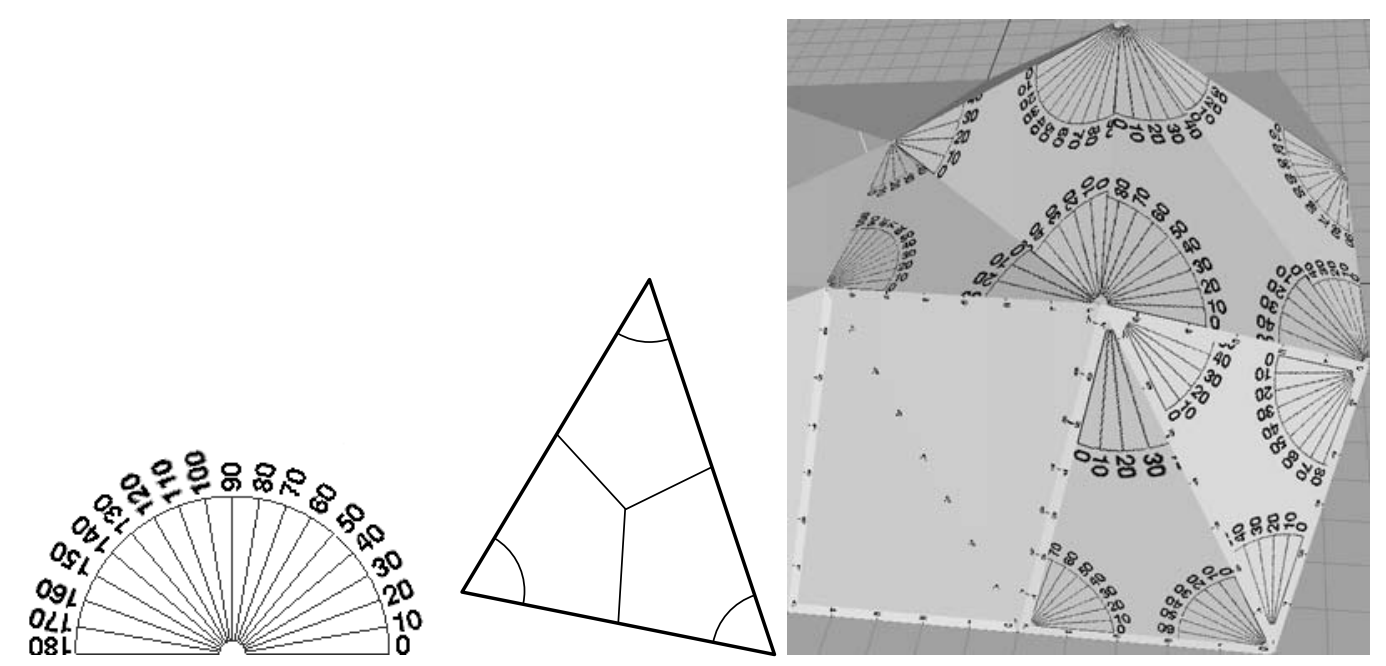

Figure 1: Left: Protractor texture applied at the vertex of each triangle.

Middle: Subdivision of a triangle into 3 subpolygons so that the arc texture can be drawn at each vertex.

Right: An example surface. Clockwise from top left: two quads with arcs applied to each vertex of each component triangle, a quad with rulers and arcs, and a quad with just rulers.

This new scheme of textured annotations on the surface of objects is quite effective at providing users with ready access to salient metrics on the surface. In an experiment which required subjects to build a simple object to precise dimensions, subjects were readily able to visually access linear measurements provided by the texture-mapped rulers. Subjects could quickly tell that they had reached the correct length.

In a surface with hundreds or thousands of quads in the mesh, each quad can be textured with rulers and arcs, making the linear dimensions of each quad visually available simultaneously without cluttering the scene with auxiliary measurement dialogs. This new texturing method eliminates input clutter and reduces output clutter.

\section{References}

[1] K P Herndon, et al. Interactive Shadows. In UIST 1992 Proceedings, pages 1-6, 1992. 